\title{
HOMOTOPY TYPE OF PATH SPACES
}

\author{
DIANE KALISH
}

(Communicated by Frederick R. Cohen)

\begin{abstract}
This paper extends the Fundamental Theorem of Morse Theory to the two endmanifold case. The theorem relates the homotopy type of the space of paths connecting two submanifolds of a Riemannian manifold to the critical points of the energy function defined on this path space. Use of the author's formulation of the Morse index Theorem in this setting allows for a simple computation of the homotopy type, and several specific examples are worked out.
\end{abstract}

\section{INTRODUCTION}

The Fundamental Theorem of Morse Theory relates the homotopy type of the space of paths $\Omega(M ; p, q)$ connecting two points $p$ and $q$ of a manifold $M$ to the critical points of the energy function $E$ defined on $\Omega(M ; p, q)$. This paper extends that theorem to $\Omega(M ; P, Q)$, the space of paths connecting two submanifolds $P$ and $Q$ in an ambient manifold $M$. As in the former case, the homotopy type of $\Omega(M ; P, Q)$ is a countable CW-complex consisting of cells of dimensions corresponding to the indices of the critical points of $E$ that are now geodesics orthogonal to $P$ and $Q$. The proof follows the one given in J. Milnor's "Morse Theory" [6, pp. 88-95], and in $\S 3$ the needed modifications are given.

In the two point case, the Morse Index Theorem yields the index of an extremal as the number of conjugate points along the geodesic. In the traditional extensions of this theorem to the two endmanifold case $[1,3,7]$, the index of the extremal has not been computed so simply from the behavior of the Jacobi fields along the geodesic. However, use of the author's formulation in the extended setting [5] does allow for a computation from such behavior (i.e., focal points) together with a boundary term at the end of the geodesic. Thus, the usual difficulties in determining the index are circumvented and so the homotopy type of $\Omega(M ; P, Q)$ can be calculated. In $\S 4$ several examples are worked out.

This paper constitutes a portion of the author's Ph.D. dissertation [4]. I would like to thank and express my gratitude to my thesis advisor, E. Feldman,

Received by the editors March 30, 1989 and, in revised form, December 21, 1990.

1991 Mathematics Subject Classification. Primary 58B05, 58E05, 58E10, 55P35.

Research for this paper was partially supported by The School of Science and Mathematics of William Paterson College under the Governor's Challenge Grant. 
for his guidance, continuing encouragement, and generosity in sharing his time and many valuable suggestions. I would also like to thank I. Chavel, D. Chess, and $\mathrm{A}$. Vasquez for engaging in many helpful discussions.

\section{Preliminaries}

$M$ is a complete Riemannian manifold of dimension $d$ with the Levi-Civita connection. $P$ and $Q$ are two submanifolds of $M$ whose dimensions are less than $d$. Let $\Omega(M ; P, Q)=\Omega$ be the space of continuous piecewise smooth paths $w:[0, T] \rightarrow M$ joining $P$ and $Q$. The energy function $E: \Omega \rightarrow \mathbf{R}$ is given by $E(w)=\int_{0}^{T}\|d w / d t\|^{2} d t$. A critical point of $E$ is a geodesic $\gamma \in \Omega$, which is orthogonal to $P$ and $Q$ at its end points.

Consider $H$, the set of all continuous piecewise smooth vector fields along and orthogonal to a critical point $\gamma$, whose values at the end points of $\gamma$ are in $T P_{\gamma(0)}$ and $T Q_{\gamma(T)}$. If $E_{1}, \ldots, E_{d-1}$ are parallel orthonormal vector fields along and orthogonal to $\gamma$, then an element $X$ in $H$ can be written as $X(t)=\sum_{i=1}^{d-1} f_{i}(t) E_{i}(t) ; X(0) \in T P_{\gamma(0)}, X(T) \in T Q_{\gamma(T)}$, and $f_{i}[0, T] \rightarrow \mathbf{R}$ are continuous piecewise smooth functions.

A symmetric bilinear functional $I: H \times H \rightarrow \mathbf{R}$, called the Morse Index Form, is defined by

$$
\begin{aligned}
I(X, Y)= & \int_{0}^{T}\left\langle R X-X^{\prime \prime}, Y\right\rangle d t+\sum_{i}\left\langle X^{\prime}\left(p_{i}^{-}\right)-X^{\prime}\left(p_{i}^{+}\right), Y\left(p_{i}\right)\right\rangle \\
& +\left\langle X^{\prime}(T)-S_{T} X(T), Y(T)\right\rangle-\left\langle X^{\prime}(0)-S_{0} X(0), Y(0)\right\rangle,
\end{aligned}
$$

where the finite number of $p_{i}$ are the points of discontinuity of $X^{\prime}(t), S_{0}$ and $S_{T}$ are the second fundamental forms of $P$ and $Q$ with respect to $\gamma^{\prime}(0)$ and $\gamma^{\prime}(T)$, and $R X=R\left(\gamma^{\prime}, X\right) \gamma^{\prime}$ is the curvature tensor of the Levi-Civita connection.

A continuous piecewise smooth vector field $J$, along and orthogonal to a critical point $\gamma$, is a $P$-Jacobi field if it satisfies the differential equation $X^{\prime \prime}$ $R X=0$, and $J(0) \in T P_{\gamma(0)}, J^{\prime}(0)-S_{0} J(0) \perp T P_{\gamma(0)}$. A point $\gamma\left(t_{i}\right)$ for $t_{i} \in(0, T]$ is a $P$-focal point if there exists a nonzero $P$-Jacobi field that vanishes at $t_{i}$, and the multiplicity of $\gamma\left(t_{i}\right)$ is the dimension of the space of all such $P$-Jacobi fields.

A symmetric bilinear functional $A$ defined on the space spanned by $P$-Jacobi fields, whose value at $t=T$ is nonzero and is contained in $T Q_{\gamma(T)}$, is given by

$$
A\left(J_{1}, J_{2}\right)=\left\langle J_{1}^{\prime}(T)-S_{T} J_{1}(T), J_{2}(T)\right\rangle,
$$

where $S_{T}$ is the second fundamental form of $Q$ at $\gamma(T)$ with respect to $\gamma^{\prime}(T)$.

The index $i$, of a symmetric bilinear functional on a vector space $V$, is the maximum dimension of a subspace of $V$ on which the functional is negative definite.

The Morse Index Theorem in the two endmanifold case can now be stated as follows.

Theorem. Assuming $\gamma(T)$ is not a P-focal point, the index of $I$ is equal to the sum of the number of $P$-focal points $\gamma(t), t \in(0, T)$-each such P-focal point counted with its multiplicity and the index of $A$.

For the proof see [5]. 
In the next section we give the definitions and modifications needed to extend the Fundamental Theorem of Morse Theory to the two endmanifold case.

\section{The Fundamental Theorem of Morse Theory}

Let $\Omega^{*}=\Omega^{*}(M ; P, Q)$ be the space of all continuous paths $w:[0,1] \rightarrow M$ joining $P$ and $Q$ in the compact open topology. This topology is induced by the metric

$$
d^{*}\left(w_{1}, w_{2}\right)=\max _{t} \rho\left(w_{1}(t), w_{2}(t)\right)
$$

where $\rho$ denotes the topological metric on $M$ coming from its Riemannian metric.

Let $\Omega=\Omega(M ; P, Q)$ be the space of all continuous piecewise smooth paths $w:[0,1] \rightarrow M$ joining $P$ and $Q$ in the topology induced by the metric

$$
d\left(w_{1}, w_{2}\right)=d^{*}\left(w_{1}, w_{2}\right)+\left\{\int_{0}^{1}\left(\frac{d s_{1}}{d t}-\frac{d s_{2}}{d t}\right)^{2} d t\right\}^{1 / 2},
$$

where $w_{1}, w_{2} \in \Omega$ with arc lengths $s_{1}(t)$ and $s_{2}(t)$.

Definition. Two submanifolds $P$ and $Q$ are said to be conjugate along a geodesic $\gamma$ orthogonal to $P$ and $Q$, if there exists a nonzero $P$-Jacobi field along $\gamma$ that is also a $Q$-Jacobi field. A $Q$-Jacobi field is defined analogously to a $P$-Jacobi field. It can be shown that a critical point $\gamma$ of the energy function $E$ is degenerate if and only if $P$ and $Q$ are conjugate along $\gamma$ [2, p. 221].

Theorem (Fundamental Theorem of Morse Theory in the two endmanifold case). Let $M$ be a complete Riemannian manifold with $P$ and $Q$ as two closed and bounded submanifolds of $M$ that are not conjugate along any geodesic orthogonal to $P$ and $Q$. Then $\Omega$ or $\Omega^{*}$ has the homotopy type of a countable $C W$-complex that contains one cell of dimension $\lambda$ for each geodesic orthogonal to $P$ and $Q$ of index $\lambda$.

The proof given in Milnor [6, pp. 88-95] is equally valid in this setting with the following modifications.

(1) A subdivision $0=t_{0}<t_{1}<\cdots t_{n}=1$ of $[0,1]$ is chosen. We define $\Omega\left(t_{0}, \ldots, t_{n}\right)$ as the subspace of $\Omega$ consisting of paths $w:[0,1] \rightarrow M$ such that:

(a) $w(0) \in P, w(1) \in Q$;

(b) $w \mid\left[t_{i-1}, t_{i}\right]$ is a geodesic for $i=2, \ldots, n-1$; and

(c) $\left.w\right|_{\left[0, t_{1}\right]}$ and $\left.w\right|_{\left[t_{n-1}, 1\right]}$ are geodesics orthogonal to $P$ and $Q$, respectively.

(2) $S$ denotes the set $\{x \in M: D(x, P) \leq \sqrt{c}\}$, where $D$ is the distance from a point $x$ to the submanifold $P$.

(3) $S$ is compact, and so we can choose an $\varepsilon>0$ as the minimum of $\bar{\varepsilon}, r_{1}$, $r_{2}$ where:

(a) $\bar{\varepsilon}>0$ : a positive real number that guarantees a unique geodesic from $x$ to $y$ of length less than $\bar{\varepsilon}$ whenever $\rho(x, y)<\bar{\varepsilon}$.

(b) $r_{1}>0$ : let $\perp(P)$ be the normal bundle of $P$ and let $\perp_{r}(P) \subset \perp(P)$ be the tubular neighborhood of $\perp(P)$ with radius $r$. There exists an $r_{1}>0$ such that the exponential function maps $\perp_{r_{1}}(P)$ diffeomorphically onto $M$ with the 
property that all points $p$ in the image are joined to $P$ by unique geodesics that minimize distance to $P$, are orthogonal to $P$, and depend differentiably on $p$.

(c) $r_{2}>0: r_{2}$ is chosen similarly with respect to $Q$. $\varepsilon^{2} / c$.

We choose the subdivision in modification 1 so that each $t_{i}-t_{i-1}$ is less than

(4) $B$ is the set of paths in $\Omega\left(t_{0}, \ldots, t_{n}\right)$ of energy less than $c>0$. Then $B$ is homeomorphic to the open subset of the $(n-1)$-fold product $M \times \cdots \times M$ given by the set of all $\left\{p_{1}, \ldots, p_{n-1}\right\} \in S \times \cdots \times S$, such that

$$
\frac{D\left(p_{1}, P\right)^{2}}{t_{1}}+\frac{\sum_{i=2}^{n-1} \rho\left(p_{i-1}, p_{i}\right)^{2}}{t_{i}-t_{i-1}}+\frac{D\left(p_{n-1}, Q\right)^{2}}{t_{n}-t_{n-1}}<c .
$$

(5) A retraction $r$ from the set of paths in $\Omega$ of energy less than $c$ into $B$ is defined as follows. Let $r(w)$ denote the unique broken geodesic in $B$ such that $\left.r(w)\right|_{\left[0, t_{1}\right]}$ and $\left.r(w)\right|_{\left[t_{n-1}, 1\right]}$ is the unique shortest geodesic of length $<\varepsilon$ joining $P$ to $w\left(t_{1}\right)$ and $w\left(t_{n-1}\right)$ to $Q$, respectively; while each $\left.r(w)\right|_{\left[t_{i-1}, t_{1}\right]}$ is the unique geodesic of length $<\varepsilon$ from $w\left(t_{i-1}\right)$ to $w\left(t_{i}\right)$ for $i=2, \ldots, n-1$.

(6) Let $E^{\prime}: B \rightarrow \mathbf{R}$ denote the restriction of $E$ to $B$. The critical points of $E$ are the unbroken geodesics that are orthogonal to $P$ and $Q$ and so lie in the submanifold $B$. Using the first variation formula, the critical points of $E^{\prime}$ are precisely the unbroken geodesics orthogonal to $P$ and $Q$.

(7) Consider the tangent space $T B_{\gamma}$ to the manifold $B$ at a geodesic $\gamma$ orthogonal to $P$ and $Q$. This will be identified with the space $K$, defined as follows.

Definition. Let $K$ be the space of vector fields $Y$ along $\gamma$ such that $\left.Y\right|_{\left[0, t_{1}\right]}$ is a $P$-Jacobi field, $\left.Y\right|_{\left[t_{i-1}, t_{i}\right]}$ is a Jacobi field for $i=2, \ldots, n-1$, and $\left.Y\right|_{\left[t_{n-1}, 1\right]}$ is a $Q$-Jacobi field. This identification can be justified as follows. Let $\bar{\alpha}(-\varepsilon, \varepsilon) \rightarrow B$ be any variation of $\gamma$ through broken geodesics in $B$. Then the corresponding vector field $(\partial \alpha / \partial u)(0, t)$ along $\gamma$ is a broken Jacobi field in $K$. The index of $I$ at $\gamma$ is equal to the index of $\left.I\right|_{K}=E_{* *}^{\prime}$, where $E_{* *}^{\prime}$ is the Hessian of $E^{\prime}$ [2, p. 232].

With these modifications the following theorems hold.

Theorem 1. Let $M$ be a complete Riemannian manifold, and let $c$ be a fixed positive number such that $\Omega^{c}$, the set of paths in $\Omega$ of energy less than or equal to $c$, is not the null set. Then for all sufficiently fine subdivisions $\left(t_{0}, \ldots, t_{n}\right)$ of $[0,1]$, the set $B$ can be given the structure of a smooth finite-dimensional manifold (see modification (4)).

Theorem 2. $E^{\prime}: B \rightarrow \mathbf{R}$ is smooth. Furthermore, for each $a<c$ the set $B^{a}=$ $\left(E^{\prime}\right)^{-1}[0, a]$ is compact and is a deformation retract of the corresponding set $\Omega^{a}$. (Similarly, $B$ is a deformation retract of Int $\Omega^{c}$.) The critical points of $E^{\prime}$ are precisely the same as the critical points of $E$ in Int $\Omega^{c}$; namely, the unbroken geodesics, from $P$ to $Q$, that are orthogonal to $P$ and $Q$, of length less than $\sqrt{c}$. The index of the Hessian $E_{* *}^{\prime}$ at each such critical point $\gamma$ is equal to the index of $I$ at $\gamma$.

Theorem 3. Let $M$ be a complete Riemannian manifold, and let $P$ and $Q$ be two closed and bounded submanifolds that are not conjugate along any geodesic 
orthogonal to $P$ and $Q$ of length $\leq \sqrt{a}$. Then $\Omega^{a}$ has the homotopy type of a finite $C W$-complex with one cell of dimension $\lambda$ for each geodesic orthogonal to $P$ and $Q$ in $\Omega^{a}$ at which $I$ has index $\lambda$.

Theorem 4. The map $i: \Omega \rightarrow \Omega^{*}$ is a homotopy equivalence.

These four theorems yield the Fundamental Theorem of Morse Theory in the two endmanifold case. For further details of the proof, see [4].

\section{EXAMPLES}

Example 1. The homotopy type of the space of paths joining two nonintersecting circles on the 2-sphere.

Let $M$ be the unit 2-sphere, $S^{2} ; P=S^{1} ; Q=S^{1}$. Consider the circles placed on $S^{2}$ as illustrated in Figure 1.

Let $\gamma(t)$ be the geodesic whose points lie on the great circle through $N$ and $B$ and whose direction is clockwise, so that $\gamma(0)=N ; \gamma\left(\frac{\pi}{6}\right)=B$, etc.

Let $E(t)$ be a unit parallel vector field along the geodesic $\gamma(t)$ such that $E(t) \in M_{\gamma(t)}$ and $E(t) \perp \gamma^{\prime}(t)$.

Let $J(t)=(\sin t) E(t)$ be a Jacobi field along $\gamma(t)$. The first four geodesics from $P$ to $Q$ that are orthogonal to $P$ and $Q$ are listed in order of increasing length:

$$
\begin{aligned}
& \gamma_{1} ; \gamma(t) \text { for } \frac{\pi}{6} \leq t \leq \frac{2}{3} \pi \text { along } B C ; \\
& \gamma_{2} ; \gamma(t) \text { for } \frac{\pi}{6} \leq t \leq \frac{7}{9} \pi \text { along } B D ; \\
& \gamma_{3} ; \gamma(t) \text { for }-\frac{\pi}{6} \leq t \leq \frac{2}{3} \pi \text { along } A C ; \\
& \gamma_{4} ; \gamma(t) \text { for }-\frac{\pi}{6} \leq t \leq \frac{7}{9} \pi \text { along } A D .
\end{aligned}
$$

For a geodesic $\gamma$, orthogonal to $P$ and $Q$ at its end points, where $\gamma(\bar{t}) \in Q$, $A(J)$ is computed as follows.

Let $\Theta \in(0, \pi / 2]$ be the length of arc from the center of the circle $Q$ on $S^{2}$ to the circumference of $Q$. Then $K_{Q}$, the geodesic curvature of $Q$, is equal

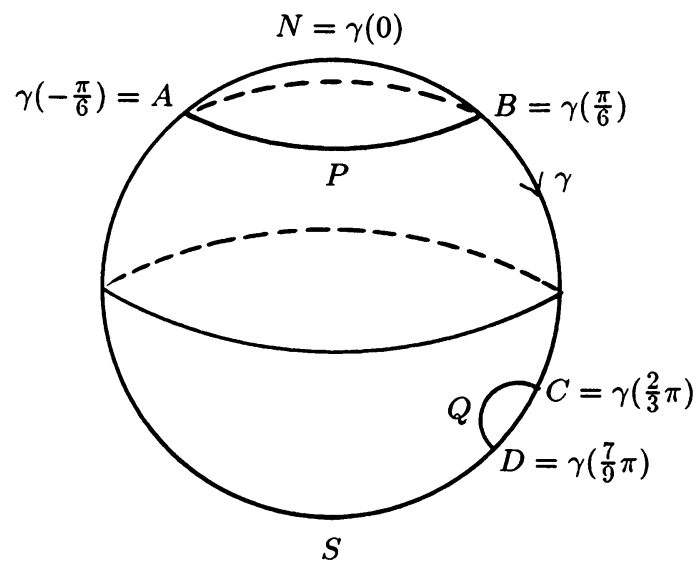

FIGURE 1 


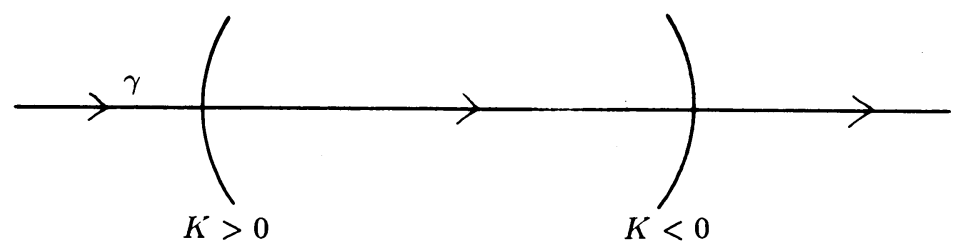

FigURE 2

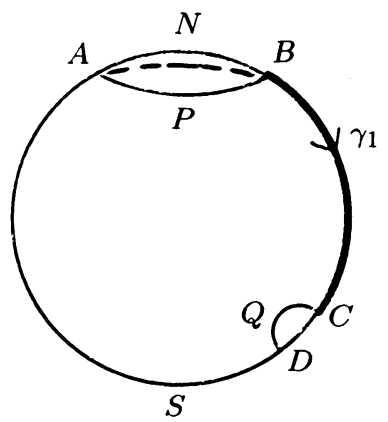

FIGURE 3

to $\cot \Theta . K_{Q}$ is positive (negative) when the circle $Q$ is bending away (towards) the geodesic as the geodesic approaches $Q$, as shown in Figure 2 .

For the $P$-Jacobi field $J(t)=(\sin t) E(t)$ the computation of $A$ in the formula for the index form is

$$
A(J)=\left.\left\langle J^{\prime}-S_{Q} J, J\right\rangle\right|_{t=\bar{t}}=\left.\left\langle J^{\prime}+K_{Q} J, J\right\rangle\right|_{t=\bar{t}}=\left(\cos \bar{t}+K_{Q} \sin \bar{t}\right)(\sin \bar{t}) .
$$

Let $i\left(\gamma_{j}\right)$ denote the index of $I$ for the geodesic $\gamma_{j}$ and let $i(A)$ denote the index of $A$ for $\gamma_{j}$.

The computations of the indices of the geodesics are as follows.

The index of the geodesic $\gamma_{1} ; B C$ (see Figure 3). The space of $P$-Jacobi fields is spanned by $J(t)$ for $\frac{\pi}{6} \leq t \leq \frac{2}{3} \pi$. That $J$ is a $P$-Jacobi field follows from

$$
\begin{aligned}
\left.\left\langle J^{\prime}-S p J, J\right\rangle\right|_{\pi / 6} & =\left(\cos \frac{\pi}{6}+K_{P} \sin \frac{\pi}{6}\right)\left(\sin \frac{\pi}{6}\right) \\
& =\left(\cos \frac{\pi}{6}+\left(-\cot \frac{\pi}{6}\right)\left(\sin \frac{\pi}{6}\right)\right)\left(\sin \frac{\pi}{6}\right)=0 .
\end{aligned}
$$

This shows $\left.\left(J^{\prime}-S p J\right)\right|_{\pi / 6} \perp P_{\gamma(\pi / 6)}$.

$$
\begin{aligned}
A\left(J\left(\frac{2}{3} \pi\right)\right) & =\left(\cos \frac{2}{3} \pi+K_{Q} \sin \frac{2}{3} \pi\right)\left(\sin \frac{2}{3} \pi\right) \\
& \approx(-.5+(5.7)(.8))(.8)>0 \quad\left[\cot \frac{\pi}{18} \cong 5.7\right] .
\end{aligned}
$$

Since $A$ is positive on the span of the $P$-Jacobi field (there is only one in this case), we have $i(A)=0$. The $P$-focal points occur where $J(t)$ vanishes, which will be at the north and south poles when $t=n \pi, n=0,1,2, \ldots$; and the multiplicity at each $P$-focal point is 1 . Therefore, $J$ has no $P$-focal points along $\gamma_{1}$. This yields

$$
i\left(\gamma_{1}\right)=(\text { number of } P \text { focal points with multiplicity })+i(A)=0 \text {. }
$$


Each time we extend $\gamma_{1}$ a complete revolution, we obtain another geodesic joining $P$ and $Q$ that is orthogonal to $P$ and $Q$, and for each complete revolution we pick up two $P$-focal points. We will denote the geodesics and their extensions by $\gamma_{1}=n(2 \pi)$ for $n=0,1,2, \ldots$. The boundary term at $Q$ remains the same for all these geodesics. Therefore, we have

$$
i\left(\gamma_{1}+n(2 \pi)\right)=2 n+i(A)=2 n .
$$

The index of the geodesic $\gamma_{2} ; B D$ (see Figure 4). A P-Jacobi field along $\gamma_{2}$ is given by $J(t)$ for $\frac{\pi}{6} \leq t \leq \frac{7}{9} \pi$.

$$
\begin{aligned}
A\left(J\left(\frac{7}{9} \pi\right)\right) & =\left(\cos \frac{7}{9} \pi+K_{Q} \sin \frac{7}{9} \pi\right)\left(\sin \frac{7}{9} \pi\right) \\
& \approx(-.8+(-5.7)(.6))(.6)<0 .
\end{aligned}
$$

So $i(A)=1$, and there are no $P$-focal points. We have

$$
i\left(\gamma_{2}\right)=0+1=1, \quad i\left(\gamma_{2}+n(2 \pi)\right)=2 n+1 .
$$

The index of the geodesic $\gamma_{3} ; A C$ (see Figure 5). A $P$-Jacobi field along $\gamma_{3}$ is given by $J(t)$ for $-\frac{\pi}{6} \leq t \leq \frac{2}{3} \pi . J$ is a $P$-Jacobi field and $A\left(J\left(\frac{2}{3} \pi\right)\right)>0$ from the calculation for $\gamma_{1}$, so we get $i(A)=0$. There is one $P$-focal point that occurs at the north pole. We have $i\left(\gamma_{3}\right)=1+0=1$ and $i\left(\gamma_{3}+n(2 \pi)\right)=2 n+1$.

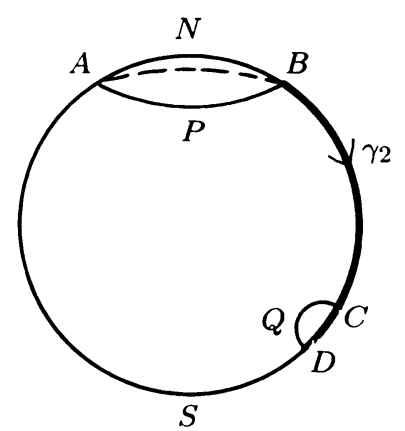

FIGURE 4

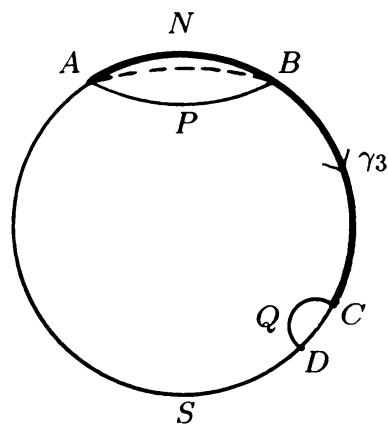

FIGURE 5 


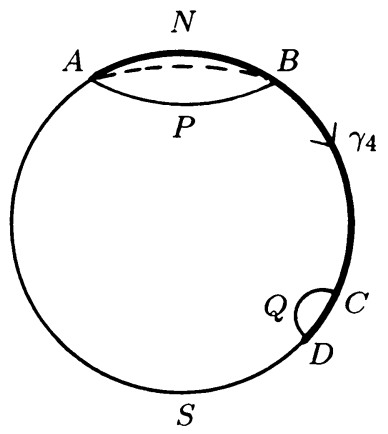

FIGURE 6

The index of the geodesic $\gamma_{4} ; A D$ (see Figure 6). A $P$-Jacobi field is given by $J(t)$ of $-\frac{\pi}{6} \leq t \leq \frac{7}{9} \pi . A\left(J\left(\frac{7}{9}\right)\right)<0$ from the calculation for $\gamma_{2}$, so we get $i(A)=1$. There is one $P$-focal point at the north pole. So we have $i\left(\gamma_{4}\right)=1+1=2$ and $i\left(\gamma_{4}+n(2 \pi)\right)=2 n+2$. For the calculation of the next four orthogonal geodesics we redefine $\gamma(t), E(t)$, and $J(t)$. Let $\gamma(t)$ be the geodesic whose points lie on the great circle through $N$ and $A$ and whose direction is counterclockwise, so that $\gamma(0)=N, \gamma\left(\frac{\pi}{6}\right)=A$, etc. Let $E(t)$ be a unit parallel vector field along $\gamma(t)$ such that $E(t) \in M_{\gamma(t)}$ and $E(t) \perp \gamma^{\prime}(t)$. Let $J(t)=(\sin t) E(t)$. The next four orthogonal geodesics from $P$ to $Q$ listed in order of increasing length, with the negative sign indicating counterclockwise direction, are $\gamma_{5}=-A D, \gamma_{6}=-A C, \gamma_{7}=-B D$, and $\gamma_{8}=-B C$.

We proceed exactly as before to calculate the index of $\gamma_{5}, \gamma_{6}, \gamma_{7}$, and $\gamma_{8}$ and summarize the results below.

$\begin{array}{rlrrrl} & n=0 & n=1 & n=2 & \ldots \\ i\left(\gamma_{1}+n(2 \pi)\right) & =2 n & 0 & 2 & 4 \\ i\left(\gamma_{2}+n(2 \pi)\right)=2 n+1 & 1 & 3 & 5 \\ i\left(\gamma_{3}+n(2 \pi)\right)=2 n+1 & 1 & 3 & 5 \\ i\left(\gamma_{4}+n(2 \pi)\right) & =2 n+2 & 2 & 4 & 6 \\ i\left(\gamma_{5}+n(2 \pi)\right) & =2 n+1 & 1 & 3 & 5 \\ i\left(\gamma_{6}+n(2 \pi)\right) & =2 n+2 & 2 & 4 & 6 \\ i\left(\gamma_{7}+n(2 \pi)\right) & =2 n+2 & 2 & 4 & 6 \\ i\left(\gamma_{8}+n(2 \pi)\right) & =2 n+3 & 3 & 5 & 7\end{array}$

Therefore, the homotopy type of the space of paths joining two nonintersecting circles on $S^{2}$ is the same as a countable CW-complex given by

$$
\begin{aligned}
& e^{0} \cup e^{1} \cup e^{1} \cup e^{2} \cup e^{1} \cup e^{2} \cup e^{2} \cup e^{3} \\
& \quad \cup e^{2} \cup e^{3} \cup e^{3} \cup e^{4} \cup e^{3} \cup e^{4} \cup e^{4} \cup e^{5} \cup e^{4} \cup e^{5} \cup \cdots, \text { etc. }
\end{aligned}
$$

Example 2. The homotopy type of the space of paths joining two interesting circles on the 2-sphere $\left(M=\right.$ unit 2-sphere, $\left.P=S^{1}, Q=S^{1}\right)$. 


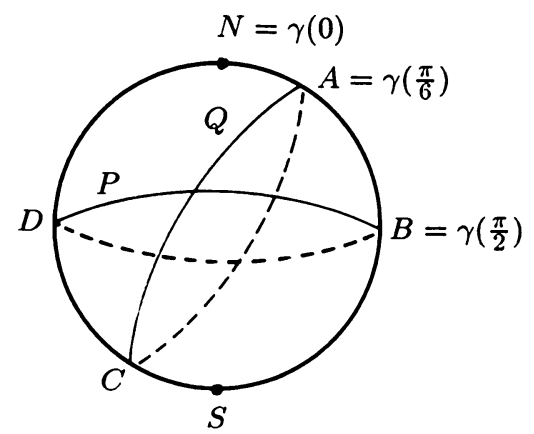

FIGURE 7

Consider the great circles placed on $S^{2}$ as illustrated in Figure 7.

Choosing the appropriate $\gamma(t)$ as in Example 1, and noting that $K_{P}=K_{Q}=$ 0 , we obtain $A(J)=(\cos \bar{t})(\sin \bar{t})$. This time there are ten geodesics to consider. The first two $\left(\gamma_{1}, \gamma_{2}\right)$ are the points of intersection of $P$ and $Q$. The results found below are listed for the geodesics in order of increasing lengths.

$$
\begin{array}{ll}
\gamma_{1}=\text { point } & i\left(\gamma_{1}+n(2 \pi)\right)=2 n \\
\gamma_{2}=\text { point } & i\left(\gamma_{2}+n(2 \pi)\right)=2 n \\
\gamma_{3}=-B A & i\left(\gamma_{3}+n(2 \pi)\right)=2 n+1 \\
\gamma_{4}=-D C & i\left(\gamma_{4}+n(2 \pi)\right)=2 n+1 \\
\gamma_{5}=D A & i\left(\gamma_{5}+n(2 \pi)\right)=2 n+1 \\
\gamma_{6}=B C & i\left(\gamma_{6}+n(2 \pi)\right)=2 n+1 \\
\gamma_{7}=-B C & i\left(\gamma_{7}+n(2 \pi)\right)=2 n+2 \\
\gamma_{8}=-D A & i\left(\gamma_{8}+n(2 \pi)\right)=2 n+2 \\
\gamma_{9}=B A & i\left(\gamma_{9}+n(2 \pi)\right)=2 n+2 \\
\gamma_{10}=D C & i\left(\gamma_{10}+n(2 \pi)\right)=2 n+2
\end{array}
$$

Therefore, the homotopy type of the space of paths joining two intersecting circles on $S^{2}$ is the same as the CW-complex

$$
\begin{aligned}
& e^{0} \cup e^{0} \cup e^{1} \cup e^{1} \cup e^{1} \cup e^{1} \cup e^{2} \cup e^{2} \cup e^{2} \cup e^{2} \\
& \cup e^{2} \cup e^{2} \cup e^{3} \cup e^{3} \cup e^{3} \cup e^{3} \cup e^{4} \cup e^{4} \cup e^{4} \cup e^{4} \cup e^{4} \cup \cdots, \text { etc. }
\end{aligned}
$$

Example 3. The homotopy type of the space of paths joining two nonintersecting $(d-1)$-spheres on a $d$-sphere $\left(M=S^{d}, P=S^{d-1}, Q=S^{d-1}\right)$ (see Figure 8 on the next page). 


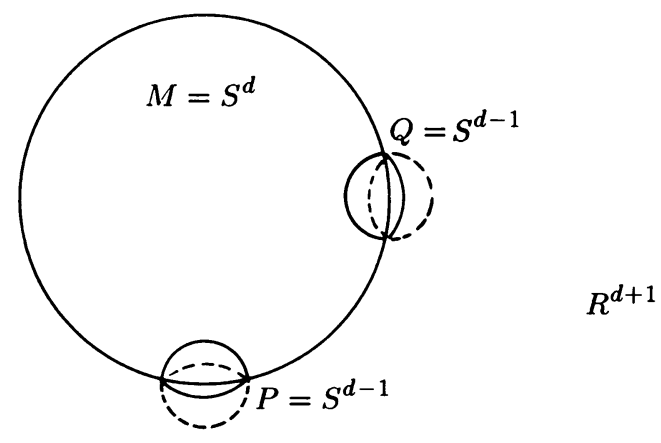

$\sigma \downarrow$ stereographic projection

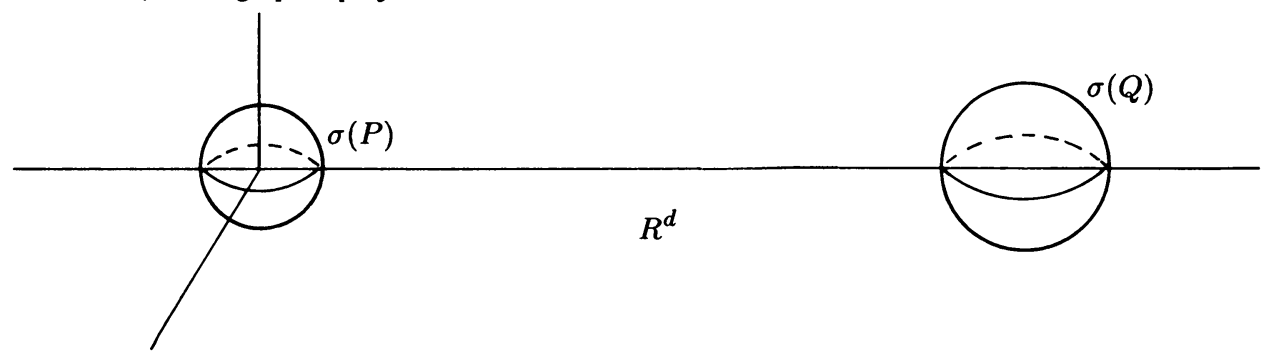

\section{FIGURE 8}

We place $S^{d}$ and $S^{d-1}$ with their centers along the $x_{d+1}$ axis and the second $S^{d-1}$ with its center not along the $x_{d+1}$ axis, as illustrated. Let $\sigma$ be a stereographic projection that sends the south pole of $S^{d}$ to the origin in $R^{d}$ and the north pole to the point at infinity. This tells us there is one great circle on $S^{d}$ that is orthogonal to $P$ and $Q$. It is the one that $\sigma$ sends into that coordinate axis that joins $\sigma(P)$ and $\sigma(Q)$ and is orthogonal to both. Along and orthogonal to this geodesic we have $(d-1)$ orthonormal parallel vector fields $E_{1}, \ldots, E_{d-1}$ and $(d-1) \quad P$-Jacobi fields given by $J_{i}(t)=(\sin t) E_{i}(t)$, $i=1, \ldots, d-1$, that are linearly independent. We will obtain the same computations as in Example 1 for the index of $A$ for each of the $P$-Jacobi fields, but this time for every complete revolution of a geodesic we will increase the number of $P$-focal points with their multiplicities by $2(d-1)$. Therefore, we will have

$$
\begin{aligned}
& i\left(\gamma_{1}+n(2 \pi)\right)=2 n(d-1), \\
& i\left(\gamma_{2}+n(2 \pi)\right)=(2 n+1)(d-1), \\
& i\left(\gamma_{3}+n(2 \pi)\right)=(2 n+1)(d-1), \\
& i\left(\gamma_{4}+n(2 \pi)\right)=(2 n+2)(d-1), \\
& i\left(\gamma_{5}+n(2 \pi)\right)=(2 n+1)(d-1), \\
& i\left(\gamma_{6}+n(2 \pi)\right)=(2 n+2)(d-1), \\
& i\left(\gamma_{7}+n(2 \pi)\right)=(2 n+2)(d-1), \\
& i\left(\gamma_{8}+n(2 \pi)\right)=(2 n+3)(d-1) .
\end{aligned}
$$

In the special case for $d=3$ we get $M=S^{3}, P=S^{2}$, and $Q=S^{2}$. The homotopy type of this path space is the same as a countable CW-complex given by 


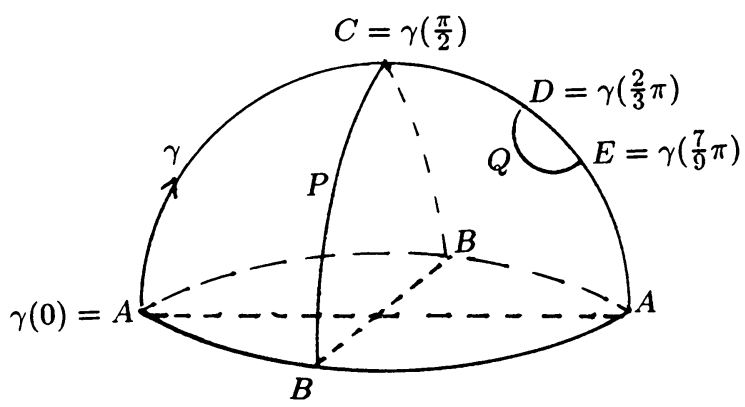

FIGURE 9

$$
\begin{aligned}
& e^{0} \cup e^{2} \cup e^{2} \cup e^{4} \cup e^{2} \cup e^{4} \cup e^{4} \cup e^{6} \\
& \cup e^{4} \cup e^{6} \cup e^{6} \cup e^{8} \cup e^{6} \cup e^{8} \cup e^{8} \cup e^{10} \cup e^{8} \cup \cdots, \text { etc. }
\end{aligned}
$$

Remark. We can compute the homology for $d>2$, and the following statement is valid [6, p. 96]. For $P, Q$ nonconjugate, let $(M ; P, Q)$ have the homotopy type of $\left(S^{d} ; S^{d-1}, s^{d-1}\right)$ for $d>2$. Then $P$ and $Q$ are joined by infinitely many geodesics orthogonal to $P$ and $Q$.

Example 4. The homotopy type of the space of paths joining real projective space $\mathbf{P}^{1}$ to a nonintersecting circle $S^{1}$ in real projective space $\mathbf{P}^{2}\left(M=\mathbf{P}^{2}, P=\right.$ $\mathbf{P}^{1}, Q=S^{1}$ ) (see Figure 9).

Let $\gamma$ be the geodesic whose points lie along the great arc that passes through $A$ and $C$ such that $\gamma(0)=A, \gamma\left(\frac{\pi}{2}\right)=C$, and $\gamma\left(\frac{2}{3} \pi\right)=D$. Let $E$ be a unit parallel vector field along $\gamma$ and orthogonal to $\gamma$ such that $E(t) \in \mathbf{T} P_{\gamma(t)}^{2}$. $J(t)=(\sin t) E(t)$ is a $P$-Jacobi field.

Computing exactly as in Example 1 and noting that each time a geodesic is extended a complete revolution only one $P$-focal point is added to the index, we get the following results for the geodesics:

$$
\begin{array}{ll}
\gamma_{1} ; C D & i\left(\gamma_{1}+n \pi\right)=n \\
\gamma_{2} ; C E & i\left(\gamma_{2}+n \pi\right)=n+1 \\
\gamma_{3} ;-C E & i\left(\gamma_{3}+n \pi\right)=n+1 ; \\
\gamma_{4} ;-C D & i\left(\gamma_{4}+n \pi\right)=n+2 .
\end{array}
$$

Therefore, the homotopy type of the space of paths joining $P^{1}$ to a nonintersecting $S^{1}$ in $\mathbf{P}^{2}$ is the same as the $\mathrm{CW}$-complex given by

$$
\begin{aligned}
& e^{0} \cup e^{1} \cup e^{1} \cup e^{2} \cup e^{1} \cup e^{2} \cup e^{2} \cup e^{3} \\
& \cup e^{2} \cup e^{3} \cup e^{3} \cup e^{4} \cup e^{3} \cup e^{4} \cup \cdots, \text { etc. }
\end{aligned}
$$

Example 5. The homotopy type of the space of paths joining two nonintersecting circles in the plane $\left(M=\mathbf{R}^{2}, P=S^{1}, Q=S^{1}\right)$. 


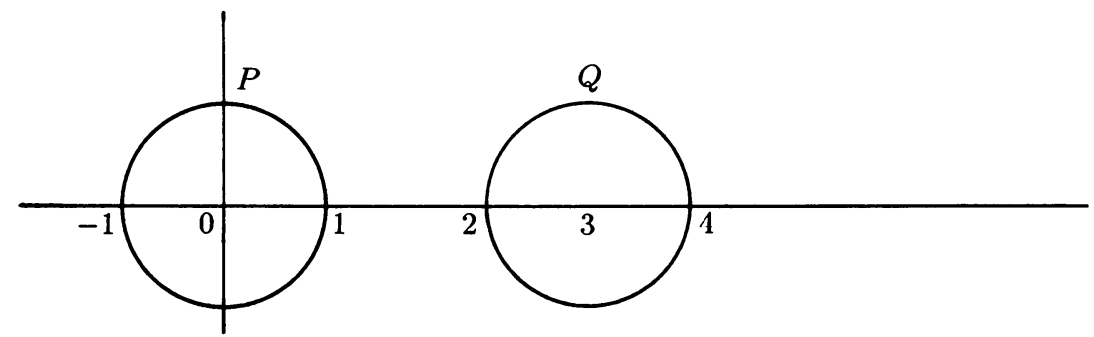

FIGURE 10

Consider the circles in Figure 10. The only geodesic orthogonal to $P$ and $Q$ is the $x$-axis. Let $E(t)$ be a unit, parallel vector field along and orthogonal to the $x$-axis. Then, $J(t)=t E(t)$ is a $P$-Jacobi field. We have

$$
\begin{aligned}
A(J(\bar{t})) & =\left.\left\langle J^{\prime}-S_{Q} J, J\right\rangle\right|_{t=\bar{t}} \\
& =\left.\left\langle E(t)+K_{Q} t E(t), t E(t)\right\rangle\right|_{t=\bar{t}}=\bar{t}+K_{Q} \bar{t}^{2} .
\end{aligned}
$$

$\gamma_{1}: \gamma_{1}$ joins 1 to $2 . A(J(2))=2+4>0$. There is no $P$-focal point, so $i\left(\gamma_{i}\right)=0$.

$\gamma_{2}: \gamma_{2}$ joins -1 to $2 . A(J(2))>0$. There is one $P$-focal point at 0 , so $i\left(\gamma_{2}\right)=1$.

$\gamma_{3}: \gamma_{3}$ joins 1 to $4 . A(J(4))=4-16<0$. There is no $P$-focal point, so $i\left(\gamma_{3}\right)=1$.

$\gamma_{4}: \gamma_{4}$ joins -1 to $4 . A(J(4))<0$. There is one $P$-focal point, so $i\left(\gamma_{4}\right)=2$.

Therefore, the homotopy type of the space is the same as the homotopy type of the finite $\mathrm{CW}$-complex $e^{0} \cup e^{1} \cup e^{1} \cup e^{2}$.

Example 6. The homotopy type of the space of paths joining two nonintersecting $S^{n}$ 's in $\mathbf{R}^{n+1}\left(M=\mathbf{R}^{n+1}, P=S^{n}, Q=S^{n}\right)$.

The situation is exactly as in Example 5, except that we now have $n P$-Jacobi fields, and so we get $e^{0} \cup e^{n} \cup e^{n} \cup e^{2 n}$.

Remark. The integral homology groups of Example 6 for $n>1$ are

$$
\begin{aligned}
H_{0} & =\mathbb{Z}, & & H_{n}=\mathbb{Z} \oplus \mathbb{Z}, \\
H_{2 n} & =\mathbb{Z}, & & H_{i}=0, \quad i \neq 0, n, 2 n .
\end{aligned}
$$

\section{REFERENCES}

1. W. Ambrose, The index theorem in Riemannian geometry, Ann. of Math. (2) 73 (1961), 49-86.

2. R. Bishop and R. Crittenden, Geometry of manifolds, Pure Appl. Math., vol. 15, Academic Press, New York, 1964.

3. J. Bolton, The Morse index theorem in the case of two variables endpoints, J. Differential Geom. 12 (1977), 567-581.

4. D. Kalish, Aspects of Morse theory, doctoral dissertation, CUNY, 1984.

5. $\ldots$, The Morse index theorem where the ends are submanifolds, Trans. Amer. Math. Soc.. 308 (1988), 341-348. 
6. J. Milnor, Morse theory, Ann. of Math. Stud., no. 51, Princeton Univ. Press, Princeton, NJ, 1973.

7. M. Morse, The calculus of variations in the large, Amer. Math. Soc. Colloq. Publ., vol. 18, Amer. Math. Soc., Providence, RI, 1934.

Department of Mathematics, William Paterson College, Wayne, New Jersey 07470

Graduate School of the City University of New York, New York, New York 